\title{
Quintessence from Shape Moduli
}

\author{
Marco Peloso 1 and Erich Poppitz \\ ${ }^{1}$ CITA, University of Toronto, Toronto, ON, M5S 3H8, Canada \\ ${ }^{2}$ Department of Physics, University of Toronto, Toronto, ON, M5S 1A7, Canada
}

(Dated: September 1, 2018)

\begin{abstract}
We show that shape moduli in sub-millimeter extra dimensional scenarios, addressing the gauge hierarchy problem, can dominate the energy density of the universe today. In our scenario, the volume of the extra dimensions is stabilized at a sufficiently high scale to avoid conflicts with nucleosynthesis and solar-system precision gravity experiments, while the shape moduli remain light but couple extremely weakly to brane-localized matter and easily avoid these bounds. Nonlocal effects in the bulk of the extra dimension generate a potential for the shape moduli. The potential has the right form and order of magnitude to account for the present day cosmic acceleration, in a way analogous to models of quintessence as a pseudo Nambu-Goldstone boson.
\end{abstract}

\section{INTRODUCTION AND SUMMARY}

There is compelling evidence that the universe is undergoing a stage of accelerated expansion. The original indication from supernovae data [1] is nicely confirmed by a wealth of independent results [2]. The most immediate explanation for this effect, a vacuum energy $\rho_{\Lambda} \sim(0.002 \mathrm{eV})^{4}$, is more than 120 orders of magnitude smaller than its naturally expected value $\sim M_{p}^{4}$. For this reason, it is often assumed that - due to some unknown mechanism- $\rho_{\Lambda}=0$, and that the acceleration is instead due to a new form of energy, named quintessence, whose effective equation of state $w$ is sufficiently close to the one of vacuum. Combining the WMAP measurement of the cosmic microwave background radiation anisotropies with supernovae [3] and large scale structure [4] observations, gives the upper bound $w \leq-0.7$ at $95 \%$ confidence level [2].

As for inflation [5], it is assumed that quintessence can be effectively described by a scalar field $Q$ [6] which is at present slowly rolling down some potential $V$. One may think that models of quintessence cannot be much harder to realize than inflationary ones. After all, primordial inflation lasted for more than $\sim 60$ e-folds, while the present accelerated stage only started at redshift $z \sim 0.5$ or so. Hence, the potential of quintessence does not need to be flat for a region of field values as large as in the case of the inflaton. However, the main trouble is now represented by the much lower scales involved. First, as we noted, $V \sim(0.002 \mathrm{eV})^{4}$ is required. Even worse, the slow roll condition limits the mass of quintessence to be smaller or comparable to the present Hubble parameter, $m_{Q} \lesssim H_{0} \sim 10^{-33} \mathrm{eV}$. Both these requirements make the realization of particle physics models of quintessence a particularly challenging task 7 ].

It is natural to invoke some symmetry to protect such low scales. For example, supersymmetric models have been considered [8]. However, we know supersymmetry to be broken at least at the TeV scale. Even assuming that the quintessence sector only feels this breaking through gravity typically results in too large a correction to $m_{Q}$. This imposes constraints on models of quintessence in supergravity [9]. As an alternative approach, quintessence has been identified with a pseudo Nambu-Goldstone boson [10]: the quintessence field acquires a mass due to the explicit breaking of some global symmetry. The reasons why the breaking could lead to a value of $m_{Q}$ which is naturally small are different for the different models considered.

In this work we discuss a different approach, with quintessence arising in the context of large extra dimensions. It is sometimes remarked that the scale $V^{1 / 4}$ of the present energy density of the universe is very close to the inverse size of the radii considered in (ADD) models of large extra dimensions 11]. Such scenarios are proposed to address the gauge hierarchy problem; the identification of quintessence with some "degree of freedom" of large extra dimensional models would thus relate in a unique framework the two strongest hierarchies of particle physics. In the scheme we have in mind, the volume of the extra dimensions is stabilized at early times, thus guaranteeing standard four dimensional gravity, while the role of quintessence is played by the moduli fields controlling the shape of the extra space. The

*Electronic address: peloso@cita.utoronto.ca

${ }^{\dagger}$ Electronic address: poppitz@physics.utoronto.ca 
quintessence potential is due to the Casimir energy of bulk fields. The scale of the potential is naturally related to the size of the extra dimensions and is protected from destabilizing corrections by locality and diffeomorphism invariance of the higher dimensional theory.

An analogous mechanism has been already discussed in the literature 12. However, due to the restricted class of shape deformations considered, it was concluded that it does lead to an equation of state $w \geq-1 / 3$, which is unable to account for the observed accelerated expansion. We show that a more optimistic conclusion is reached once generic deformations of the extra space are taken into account, and that indeed such a mechanism can lead to a successful model for quintessence. As we will see, the evolution of the shape moduli strongly resembles the one of quintessence as a pseudo Nambu-Goldstone boson [10]. ${ }^{1}$

In this paper, we focus on the case of two large extra dimensions compactified on a torus, where the generic shape deformations are known and where the Casimir energy can be accurately computed. Our results on the shape moduli as quintessence can be summarized as follows:

1. In a non supersymmetric context, the correct scale for quintessence is achieved for extra dimensions of size $L \sim 50 \mu \mathrm{m}$, corresponding to a fundamental scale of $\sim 7 \mathrm{TeV}$; remarkably, such a value of $L$ is expected to be soon tested in short distance gravity experiments 15 . However, there is already a stronger bound (less than one order of magnitude smaller than the value we are considering) from the necessity to avoid a too rapid cooling of supernovae [16].

2. If, instead, the bulk of the extra dimensions is supersymmetric, while supersymmetry is broken on the Standard Model brane at the fundamental scale $(\mathrm{TeV})$, the Casimir energy potential of the shape moduli acquires an additional suppression factor. We show that the suppression is precisely of the right order of magnitude for the potential to account for the late-time acceleration, once the scale $L$ is taken to be consistent with the supernovae bound.

This paper is organized as follows. In section ஹwe discuss the early time stabilization of the volume of the extra dimensions. In section 11 we study the Casimir energy responsible for the late time evolution of the shape moduli, and their role as quintessence. The case of a supersymmetric bulk is considered in section IV

\section{GENERAL SETUP AND AREA STABILIZATION}

In ADD models [1], the observed weakness of gravity, i.e. the high value for the effective four dimensional Planck mass $M_{p}$, is due to the large size of some extra dimensional space where gravity propagates. Generally, in models with extra dimensions, $M_{p}$ is proportional to the volume of the extra space. Variations of $M_{p}$ are strongly limited by the requirement of a conventional four dimensional cosmology from Primordial Nucleosynthesis, starting after about the first second of the universe, up to the present [17, 18. A massless radion (i.e. the scalar field whose expectation value determines the volume of the extra space) also modifies late time gravity. The radion couples to the trace of the energy momentum tensor of matter fields. While the coupling is naturally expected to be of gravitational strength, a further $\sim 10^{-4}-10^{-3}$ suppression is needed to fulfill precision tests of general relativity within the solar system 19. For these reasons, we discuss a mechanism where the volume of the extra space is stabilized at a sufficiently high scale. $^{2}$

Stabilization mechanisms for the extra dimensions have drawn considerable interest. The set-up discussed in [21] is particularly simple: two extra dimensions compactified on a Riemann surface $\left(S^{2}, T^{2}\right.$, etc.) are considered. The area $\mathcal{A}$ of the surface is stabilized due to an interplay between the six dimensional cosmological constant and a $U(1)$ field in the bulk. Under the effect of a negative $\Lambda_{6}$, it is energetically favored for the extra space to shrink. On the other hand, a gauge field in the extra compact space can have nonvanishing magnetic flux, which (analogous to the Dirac monopole in four dimensions [22]) is quantized in units of inverse charge, $\Phi \equiv \int d X^{4} d X^{5} \epsilon^{\mu \nu} F_{\mu \nu}=2 \pi N / e$, where $N$ is an integer and $e$ is the gauge coupling (which has inverse mass dimension). As a consequence, both the magnetic field and its energy are proportional to the inverse of the area $\mathcal{A}$, contrasting the shrinking of the extra-space due to the bulk cosmological constant. Minimizing the total energy, one finds that the area $\mathcal{A}$ is stabilized at:

$$
\langle\mathcal{A}\rangle=\frac{\mu}{\sqrt{-\Lambda_{6}}} \quad, \quad \mu \equiv \frac{\pi N}{\sqrt{2} e}
$$

\footnotetext{
${ }^{1}$ Quintessence in (small) extra dimensions and with a potential similar to the one of [10] was obtained also in [13]. In that work, the extra space is taken to be fixed, and the potential of a Wilson line of a gauge group in the extra space 14 is employed.

2 An example of the radion as quintessence is given in [20]; the specific form of the potential and kinetic terms and an accurate choice of initial conditions prevent a conflict of the radion time variation with the above bounds.
} 


$$
\Rightarrow \quad \frac{M_{p}^{2}}{2} \equiv M_{6}^{4}\langle\mathcal{A}\rangle=\frac{M_{6}^{4} \mu}{\sqrt{-\Lambda_{6}}}
$$

A large size of the extra dimensions can be achieved with a sufficiently large number of flux quanta, $N \gg 1$. Such a high value is technically natural, since $N$ can not be changed by (perturbative) quantum corrections. ${ }^{3}$

We are interested in studying compactification manifolds which have shape moduli. The simplest example is a toroidal extra dimension. If we place, for example, four branes, with tensions tuned so that each has deficit angle $\pi$, at the four fixed points of $T^{2} / Z_{2}$, we obtain a space with the topology of a sphere - a "pillow" with branes at the four corners - with a metric which has all the moduli of the torus (given by eqns. (2] 3) below). We can then apply the mechanism of [21] to achieve area stabilization.

We should note, however, that the magnetic flux/six dimensional cosmological constant configuration of [21] is not an exact solution of the equations of motion, as can be easily checked by computing the local Einstein equations in the extra dimensions: the backreaction of the magnetic field on the geometry of the extra space is neglected in [21] (since the backreaction can be considered a perturbation if the energy densities responsible for stabilization are small in units of $M_{6}$ ). We used the example studied in 21] as an illustration, since it only fixes the value of $\mathcal{A}$, leaving the other moduli of the extra space free to evolve; however, it is not immediately clear to us if this will still be the case once the backreaction on the geometry is taken into account.

We take the mechanism of [21] as a strong indication that the desired stabilization of the area can be achieved. However, this is not our main focus in this paper; hence, we will not further study this or other possible area stabilizing mechanisms here. In the rest of this work we simply postulate that such a mechanism exists, and focus on the late time evolution of the shape moduli fields.

\section{SHAPE STABILIZATION AND QUINTESSENCE}

We concentrate on the case of two extra dimensions compactified on a torus. This is the simplest example which allows for deformations of the extra space which preserve a fixed volume. One may regard this case as a prototype of the generic case in which more extra dimensions or shape moduli are present.

The torus is parameterized by its area $\mathcal{A}$ and by two real moduli fields $\tau_{1}, \tau_{2}$, defining its shape. With the area stabilized, the line interval is given by:

$$
d s^{2}=g_{\mu \nu}(x) d x^{\mu} d x^{\nu}+\gamma_{i j}(x) d y^{i} d y^{j} .
$$

The $x$ coordinates span the noncompact $3+1$ dimensional space, while $y^{i}$ are the two extra dimensional coordinates defined in the interval $y^{i} \in[0, L], L=\langle\mathcal{A}\rangle^{1 / 2}$. The four dimensional metric $g_{\mu \nu}$ is the metric of a FriedmannRobertson-Walker universe, while the metric $\gamma_{i j}$ on the torus is:

$$
\gamma_{i j}=\frac{1}{\tau_{2}}\left(\begin{array}{cc}
1 & \tau_{1} \\
\tau_{1} & |\tau|^{2}
\end{array}\right)
$$

where $\tau=\tau_{1}+i \tau_{2}$, so that $\gamma$ has determinant one. Integrating over the extra space, we obtain the following action, describing gravitational and moduli physics at scales larger than $L$ :

$$
\begin{aligned}
S_{g} & =M_{6}^{4} \int d^{6} x \sqrt{-G} R(G)= \\
& =\frac{M_{p}^{2}}{2} \int d^{4} x \sqrt{-g}\left[R(g)+\frac{g^{\mu \nu} \partial_{\mu} \tau \partial_{\nu} \bar{\tau}}{2 \tau_{2}^{2}}\right],
\end{aligned}
$$

where $M_{p}$, the (reduced) Planck mass in four dimensions, was given in eqn. (11), and $\bar{\tau}=\tau_{1}-i \tau_{2}$.

The Standard Model fields are confined on a $3+1$ dimensional brane and do not couple to the shape ${ }^{4}$ moduli $\tau$; they only probe the geometry of the compact space through the strength of the gravitational interactions set by

${ }^{3}$ One might also hope that a more natural mechanism can be employed, where large extra dimensions are achieved without exponentially large parameters. Some examples are provided in the literature, e.g. [23].

${ }^{4}$ Nonlocal effects in the bulk induce nonderivative couplings between shape moduli and brane-localized matter. However, in addition to the $1 / M_{p}$ suppression of the radion coupling to brane matter, the shape moduli coupling is further suppressed by factors of at least $1 /\left(L M_{6}\right) \sim 10^{-15}$. 
the volume. On the contrary, the Kaluza-Klein spectrum of any bulk field depends on both the volume and shape of the extra space. Thus, mass differences among KK eigenstates found in accelerator experiments would allow a reconstruction of the geometry of the extra space 24].

What is more important for us is that the dependence of the KK spectrum on the shape moduli may provide a stabilization mechanism for $\tau_{1,2}$. The Casimir energy of a real massless scalar field obeying periodic boundary conditions in $y^{1}, y^{2}$ of eqn. (2), generates a potential for the shape moduli given by:

$$
\begin{aligned}
V_{s} & =\frac{1}{L^{4}}\left[-\frac{4 \pi^{3} \tau_{2}^{3}}{945}-\frac{1}{2 \pi^{2} \tau_{2}^{2}}\left(\tau_{2}^{2} \frac{\partial^{2}}{\partial \tau_{2}^{2}}-3 \tau_{2} \frac{\partial}{\partial \tau_{2}}+3\right) \sum_{p=1}^{\infty} S_{p}\right], \\
S_{p} & \equiv \frac{1}{p^{5}} \frac{\sinh \left(2 \pi p \tau_{2}\right)}{\cosh \left(2 \pi p \tau_{2}\right)-\cos \left(2 \pi p \tau_{1}\right)},
\end{aligned}
$$

where we correct an overall $1 / 2$ factor in eqn. (61) of [25].

While $V_{s}$ is the Casimir energy computed in a background (2), with $g_{\mu \nu}$ - the Minkowski metric, it is easy to see that corrections due to the time-dependent nature of $g_{\mu \nu}$ are of order $H L$, where $H$ is the four dimensional Hubble scale. Using (5D) in a time-dependent background is then self-consistent provided $H \ll L^{-1}$, which is always true for energies below the fundamental scale of the theory. We also require that $V_{s}$ can be considered to be a perturbation of the volume stabilization mechanism, i.e. the Casimir energy density (5) should be (at least) smaller than $M_{6}^{4}$. The exponential dependence of (5) on the canonically normalized $\phi_{2}=\left(M_{p} / \sqrt{2}\right) \ln \tau_{2}$ thus limits $\phi_{2} / M_{p}<\mathcal{O}(10)$ (moreover, assuming an equally spaced probability for the initial value of $\phi_{2}$, values $\tau_{2} \sim 1$ are more probable than higher values).

It is also important to note that the Casimir potential for the moduli is a nonlocal effect in the bulk: there exist no local generally covariant expressions in $6 \mathrm{~d}$ that generate a potential for $\tau$. Thus eqn. (5) is not subject to divergent radiative corrections - all divergences are absorbed by local counterterms and renormalize the coefficients of various terms in the local action of the model. Nonlocal quantum gravity effects are also expected to be exponentially suppressed if the size of the extra dimension is larger than the fundamental gravity scale $M_{6}$. The scale of the potential (5) is thus naturally related to the size of the extra dimensions.

The contributions of other massless bulk fields are easily related to (5). For example, the contribution of bulk gravitons and massless periodic bulk Weyl fermions to the moduli potential is $V_{g}=9 V_{s}$ and $V_{\psi}=-4 V_{s}$, respectively [25]. In the following, we consider a simple extension of the Standard Model characterized by the presence of three right handed neutrinos $N$, as well as gravity. Since $N$ are uncharged under the Standard Model interactions they can be naturally thought as bulk fields [26]. Moreover, we assume that they are sufficiently light and so contribute to the Casimir energy as effectively massless fields (one can also simply postulate the existence of a number of massless bulk fermions). In this example, the total Casimir energy is given by:

$$
V=9 V_{s}-3 \times 4 V_{s}=-3 V_{s} .
$$

The shape moduli potentials (5) 6), induced by the Casimir energy, inherit important symmetries coming from the large diffeomorphisms of the extra compact space, which relate equivalent tori. For example, it is obvious from the form of $S_{p}$ that $V_{s}$ is a periodic function of $\tau_{1}$ with period one. More generally, equivalent tori are connected by $\mathrm{SL}(2, Z)$ transformations, $\tau \rightarrow(a \tau+b) /(c \tau+d)$, where $a, b, c$, and $d$ are integers obeying $a d-b c=1$. While this is less obvious from the explicit expression (5), the Casimir potential is invariant under the entire group of $\mathrm{SL}(2, Z)$ transformations; this fact considerably simplifies its study, as we see below.

Distinct tori have modular parameters $\tau$ taking values in the fundamental region $|\tau| \geq 1,-1 / 2 \leq \tau_{1}<1 / 2, \tau_{2}>0$, so it is enough to study the behavior of the Casimir energy in this region. Two values, $\tau_{A}=i$ and $\tau_{B}=\mathrm{e}^{2 \pi i / 3}$, are of special interest, since they are fixed points of the transformations $\tau \rightarrow-1 / \tau$ and $\tau \rightarrow-1 /(1+\tau)$, respectively (the $\mathcal{S}$ and $\mathcal{S} \mathcal{T}^{-1}$ generators of $\operatorname{SL}(2, Z)$ ). Hence, they correspond to extrema of the Casimir energy (15). To study their stability, we expand the potential (6) up to quadratic order (in practice, only the first few terms in the sum over $p$ in (5) are important):

$$
\begin{aligned}
& \left.L^{4} V\right|_{\tau \simeq \tau_{A}} \simeq 0.9-0.7 \tau_{1}^{2}+3.4\left(\tau_{2}-1\right)^{2} \\
& \left.L^{4} V\right|_{\tau \simeq \tau_{B}} \simeq 0.8+1.6\left(\tau_{1}+1 / 2\right)^{2}+1.6\left(\tau_{2}-\sqrt{3} / 2\right)^{2} .
\end{aligned}
$$

Thus, $\tau_{A}$ is a a saddle point of the potential, while $\tau_{B}$ is a minimum. In the following, we redefine $V(\tau) \rightarrow$ $V(\tau)-V\left(\tau_{B}\right)$ such that the minimum is at zero energy; the additional constant contribution is provided by the mechanism responsible for the cancellation of the total cosmological constant in the vacuum of the theory, as it is usually assumed in models of quintessence. In figure 1 we show a contourplot of the resulting potential, in the range 


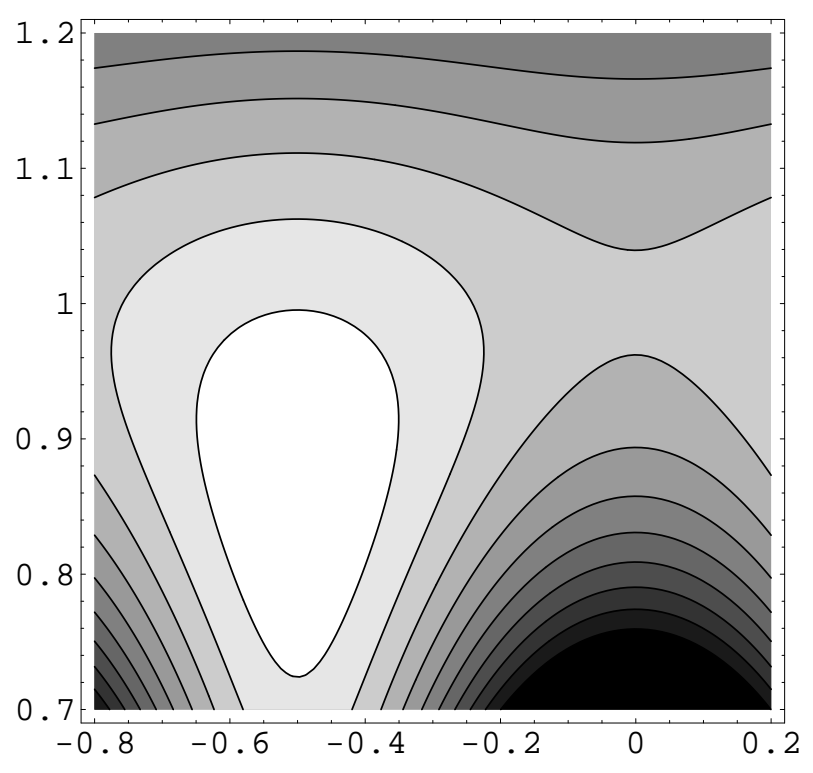

FIG. 1: Casimir potential, eqn. (6), in the $\tau_{1}-\tau_{2}$ plane. The potential is periodic in the $\tau_{1}$ direction and rises for $\tau_{2} \gg 1$ (and $\tau_{2}<1$; the two regions are physically equivalent, see text). The global minimum, $\tau_{B}=e^{2 \pi i / 3}$, is indicated in white.

of parameters of our interest. This will help illustrate the dynamics of the two moduli and their role as quintessence fields.

The situation is analogous to the one encountered in models where the role of quintessence is played by a pseudo Nambu-Goldstone boson [10] (axion) $\phi$ with lagrangian:

$$
\mathcal{L}=\frac{1}{2}\left(\partial_{\mu} \phi\right)-U \quad, \quad U=U_{0}\left(1-\cos \frac{2 \pi \phi}{f}\right) .
$$

In this case, it is assumed that $\phi$ is frozen at the maximum of the potential $U$ until very recent times. This is possible due to the friction provided by the expansion of the universe, as evident from the equation of motion $\ddot{\phi}+3 H \dot{\phi}+d U / d \phi=0$ : at early times, the contributions to the Hubble parameter $H$ from matter and radiation are high enough to prevent $\phi$ from moving. As the universe expands, $H$ drops, and eventually $\phi$ starts rolling towards the minimum of $U$. If $\phi$ is initially sufficiently close to the saddle point, the first part of its motion occurs in the slow roll regime, and a finite period of inflation - sufficient to take into account the late time acceleration of the universe - can take place.

We emphasize that, for this mechanism to work, both the scales of the potential $U_{0}$ and of the field $f$ have to acquire very precise, yet very different values. On one hand, the potential energy of $\phi$ must dominate the present energy density of the universe, leading to $U_{0} \simeq(0.002 \mathrm{eV})^{4}$. On the other hand, if we want the field to be in the slow roll regime today, rather than having settled already to the minimum of $U$, its effective mass squared $d^{2} U / d \phi^{2} \sim U_{0} / f^{2}$ has to be comparable to the present value of $H^{2}$ (this is probably the main obstacle for particle physics models of quintessence). As a consequence, $f$ cannot be taken much smaller than $M_{p}$, unless one is willing to strongly fine-tune the initial value of $\phi$ unnaturally close to the maximum of the potential $U$. It is rather remarkable that, in the proximity of the saddle point $\tau_{A}$, the potential (6) for the moduli fields is exactly of the form (8), with the two scales $U_{0}$ and $f$ precisely as required to have a workable model of quintessence.

As clear from the expansion (7), motion for $\tau \sim \tau_{A}$ initially occurs along the $\tau_{1}$ direction, where the potential $V$ has a $\cos \tau_{1}$ dependence. Since the motion of $\tau$ during the slow roll regime is rather limited, the potential experienced during the accelerated stage is effectively of the form (8) (one can verify numerically that even a simple quadratic expansion as in (7) is sufficient). Thus, the degree of fine tuning on the initial conditions is the same as the one encountered in models of Quintessence as a pseudo Nambu-Goldstone boson field [10].

The scale $f$ of the moduli fields can be easily obtained by direct inspection of their equation of motion (notice the nonstandard kinetic term in eqn. (4) $)$, and it amounts to $f \simeq 0.8 M_{p}$. Once shifted to have zero cosmological constant in the minimum, the potential is of order $V\left(\tau \simeq \tau_{A}\right) \simeq 0.1 / L^{4}$. This is of the order of the present energy density of the universe for a size of the extra dimensions $L \sim 50 \mu \mathrm{m}$. In ADD models, this corresponds to a fundamental scale of $\sim 7 \mathrm{TeV}$, reasonably close to the electroweak scale. Quite interestingly, gravity experiments give at present the 
bound $L \lesssim 150 \mu \mathrm{m}$, and are expected to lower their sensitivity to the value we are considering in the near future [15].

Despite the fact that $L \sim 50 \mu \mathrm{m}$ is compatible with present bounds from gravitational experiments, the production of a large number of light graviton Kaluza-Klein modes leads to rapid cooling of supernovae and places an upper limit on the value of the radius, $R \equiv L /(2 \pi)$. For two extra dimensions the bound is $R \leq 0.96 \mu \mathrm{m}$ [16], while our prediction from late-time domination is $R \sim 7 \mu \mathrm{m}$.

Thus, it appears that the supernovae constraint—which was not taken into account in refs. [20], [12]—-would rule out the use of shape moduli of two large extra dimensions as quintessence. ${ }^{5}$ However, as we discuss in the next section, if the bulk of the extra dimension is supersymmetric, while supersymmetry is broken on the Standard Model brane at the fundamental scale $(\mathrm{TeV})$, the Casimir energy potential of the shape moduli acquires an additional suppression factor. We will see that the suppression has just the right order of magnitude for the potential to account for the late-time acceleration, even for $L$ small enough to be consistent with the supernovae bounds.

To conclude this section, we compare our findings to the ones of [12], where late time changes of the shape of the extra space were discussed as well. Only rectangular $n$-tori were considered in [12], and an exponential potentialunable to provide late tame acceleration - was recovered. In our set-up, the particular class of shape deformations considered in [12] amounts in varying the modulus field $\tau_{2}$, with $\tau_{1}$ fixed to zero. If we do so, we also recover an exponential-like potential, for the canonically normalized field $\phi_{2} \propto \ln \tau_{2}$, cf. eqn. (5). We see, however, that the dynamics of the system does not generally proceed through fixed $\tau_{1}$. As we have discussed, it is precisely the motion along the $\tau_{1}$ coordinate which allows for a mechanism suitable for quintessence.

\section{SHAPE STABILIZATION WITH SUPERSYMMETRY IN THE BULK}

In this section, we discuss the evolution of the shape moduli fields in a supersymmetric context, where bulk fields form complete supersymmetric multiplets. If supersymmetry is exact, the contributions to the Casimir energy from bulk fermions and bosons cancel. However, supersymmetry must be broken on our brane, with mass splittings within supermultiplets $\sim \mathcal{O}(1) \mathrm{TeV}$. The supersymmetry breakdown is then communicated to fields in the bulk. We assume that the communication is of gravitational strength, giving a mass splitting within bulk supermultiplets of order $M<\mathrm{TeV}^{2} / M_{p} \sim 10^{-3} \mathrm{eV}$; this scaling is consistent with the assumption of brane-localized supersymmetry breaking, whose effect on the bulk modes should vanish in the infinite volume limit. While the precise bulk spectrum will certainly depend on the details of the model (for example, various coupling factors can occur in the above relation), we stress that our aim here is not to present a complete model of how supersymmetry breaking and its communication to the bulk occur. We are rather interested in the order of magnitude effects on the shape moduli potential.

The mass splitting within the bulk multiplets leads to a nonvanishing Casimir potential. For completeness and further use below, we give the expression for the Casimir potential of a massive bulk scalar field obeying periodic boundary conditions in $y^{1}, y^{2}$. The massless case result, eqn. (5), is replaced by:

$$
\begin{aligned}
V_{s} & =-\frac{4(M R)^{3} \tau_{2}^{3 / 2}}{L^{4}} \sum_{p=1}^{\infty} \frac{K_{3}\left(2 \pi p M R / \sqrt{\tau_{2}}\right)}{p^{3}}-\left.\frac{1}{2 \pi^{2} L^{4} \tau_{2}^{2}}\left[\sum_{p=1}^{\infty}\left(\tau_{2}^{2} \frac{\partial^{2}}{\partial \tau_{2}^{2}}-3 \tau_{2} \frac{\partial}{\partial \tau_{2}}+3\right) \tilde{S}_{p}(x)\right]\right|_{x=M R / \sqrt{\tau_{2}}}, \\
\tilde{S}_{p}(x) & \equiv \sum_{n=-\infty}^{\infty} \mathrm{e}^{-2 \pi p \tau_{2} \sqrt{n^{2}+x^{2}}} \frac{\cos \left(2 \pi p n \tau_{1}\right)}{p^{5}}
\end{aligned}
$$

where $R=L /(2 \pi)$ and $K_{3}$ is a modified Bessel function. As usual, terms that can be absorbed into local bulk or brane counterterms are omitted from (9); as discussed in the previous section, such terms are $\tau$-independent. A periodic fermion (of the same mass) would contribute an amount equal to $-4 V_{s}$.

In the case under consideration, $M R \ll 1$-recall that $R \leq \mathcal{O}(1) \mu \mathrm{m}$, while $M<10^{-3} \mathrm{eV}$-hence it is convenient to expand the potential in a power series in $M R$. The leading contribution is obtained for $M=0$ and coincides with the massless expression (5). However, the fermionic and bosonic contributions to the Casimir energy cancel for $M=0$ (exact supersymmetry in the bulk). The first nonvanishing contribution occurs due to the supermultiplet mass splitting and amounts to:

$$
\tilde{V}=\frac{(M R)^{2}}{L^{4}}\left[\frac{\pi^{3} \tau_{2}^{2}}{45}+\sum_{p=1}^{\infty} F_{p}\right]
$$

\footnotetext{
${ }^{5}$ There are also cosmological bounds which are more stringent than the value we are predicting. However, they can be more easily evaded than the one from supernovae [17, 27].
} 


$$
F\left(\tau_{1}, \tau_{2}, p\right)=-\frac{2 \pi}{p^{2}} \frac{1-\cosh \left(2 \pi p \tau_{2}\right) \cos \left(2 \pi p \tau_{1}\right)}{\left[\cosh \left(2 \pi p \tau_{2}\right)-\cos \left(2 \pi p \tau_{1}\right)\right]^{2}}+\frac{1}{\tau_{2} p^{3}} \frac{\sinh \left(2 \pi p \tau_{2}\right)}{\cosh \left(2 \pi p \tau_{2}\right)-\cos \left(2 \pi \tau_{1}\right)} .
$$

Eqn. (10) shows the contribution of a 1/4 hypermultiplet where the scalar has mass $M$ while the fermion is massless. The contributions of other multiplets with supersymmetry breaking mass splittings can be obtained from eqn. (10), as in the previous section.

Each term in the small- $M R$ expansion of (9) has the same $\operatorname{SL}(2, Z)$ symmetry as eqn. (5). In particular, the two points $\tau_{A, B}$ are also extrema of the potential term (10). The quadratic expansions around these points are:

$$
\begin{aligned}
&\left.L^{4} \tilde{V}\right|_{\tau \simeq \tau_{A}} \simeq(M R)^{2}\left[1.92-0.5 \tau_{1}^{2}+2.5\left(\tau_{2}-1\right)^{2}\right] \\
&\left.L^{4} \tilde{V}\right|_{\tau \simeq \tau_{B}} \simeq(M R)^{2}\left[1.84+1.2\left(\tau_{1}+1 / 2\right)^{2}+1.2\left(\tau_{2}-\sqrt{3} / 2\right)^{2}\right]
\end{aligned}
$$

Thus the point $\tau_{A}=i$ is a saddle point, while $\tau_{B}=e^{2 \pi i / 3}$ is the absolute minimum of the potential. To obtain shape moduli stabilization, we then require that the contributions of bulk hypermultiplets (the supersymmetry analogue of the bulk $N$-fields of the previous section) dominate the contributions of the graviton supermultiplet.

The situation is thus completely analogous to the one discussed in the previous section, with one important difference: the scale of the potential, after subtracting the value at the minimum, is $\left(0.1 / L^{4}\right)(M R)^{2}$, and is suppressed by the additional $(M R)^{2}$ factor, due to the fact that it vanishes in the supersymmetric limit. Because of this suppression, the Casimir energy can now be of the order of the present energy density of the universe for an extra dimension of size $L$ smaller than the $L=50 \mu \mathrm{m}$ we found in the previous section. For example, we can now fix $L \sim 6 \mu \mathrm{m}$ at its upper allowed limit from 16. The Casimir energy is then of the correct scale for $M \sim 2 \cdot 10^{-3}$ eV; since $V$ scales as $M^{2} / L^{2}$ this is also an upper value for $M$ (we absorb in $M$ various model-dependent factors of order one, coming from, e.g., the number of bulk multiplets). As discussed in the beginning of this section, for a supersymmetry breaking scale on the brane of order $\mathrm{TeV}$, communicated gravitationally to the bulk, a supersymmetry breaking splitting of order $10^{-3}$ $\mathrm{eV}$ in the bulk is a natural value. Thus, we conclude that shape moduli in large $(L \sim \mathcal{O}(1) \mu \mathrm{m})$ extra dimensions with $\mathrm{TeV}$-scale brane-localized supersymmetry breaking naturally provide a viable candidate for quintessence.

\section{Acknowledgements}

We thank Keith Dienes, Tony Gherghetta, Joel Giedt, and Bob Holdom for useful discussions.

[1] A. G. Riess et al., Astron. J. 116, 1009 (1998); S. Perlmutter et al., Astrophys. J. 517, 565 (1999).

[2] D. N. Spergel et al., arXiv:astro-ph/0302209

[3] A. G. Riess et al., Astrophys. J. 560, 49 (2001).

[4] M. Colless et al., arXiv:astro-ph/0106498

[5] A. D. Linde, Particle Physics and Inflationary Cosmology (Harwood, Chur, Switzerland, 1990).

[6] B. Ratra and P. J. Peebles, Phys. Rev. D 37, 3406 (1988); R. R. Caldwell, R. Dave and P. J. Steinhardt, Phys. Rev. Lett. 80, $1582(1998)$.

[7] C. F. Kolda and D. H. Lyth, Phys. Lett. B 458, 197 (1999).

[8] P. Binetruy, Phys. Rev. D 60, 063502 (1999); A. Masiero, M. Pietroni and F. Rosati, Phys. Rev. D 61, 023504 (2000).

[9] P. Brax and J. Martin, Phys. Lett. B 468, 40 (1999); P. Brax and J. Martin, Phys. Rev. D 61, 103502 (2000); E. J. Copeland, N. J. Nunes and F. Rosati, Phys. Rev. D 62, 123503 (2000).

[10] J. A. Frieman, C. T. Hill, A. Stebbins and I. Waga, Phys. Rev. Lett. 75, 2077 (1995); S. M. Carroll, Phys. Rev. Lett. 81, 3067 (1998); J. E. Kim, JHEP 9905, 022 (1999); K. Choi, Phys. Rev. D 62, 043509 (2000); J. E. Kim, JHEP 0006, 016 (2000); Y. Nomura, T. Watari and T. Yanagida, Phys. Lett. B 484, 103 (2000); C. T. Hill and A. K. Leibovich, Phys. Rev. D 66, 075010 (2002) J. E. Kim and H. P. Nilles, Phys. Lett. B 553 (2003) 1.

[11] N. Arkani-Hamed, S. Dimopoulos and G. R. Dvali, Phys. Lett. B 429, 263 (1998); I. Antoniadis, N. Arkani-Hamed, S. Dimopoulos and G. R. Dvali, Phys. Lett. B 436, 257 (1998).

[12] M. Pietroni, Phys. Rev. D 67, 103523 (2003).

[13] L. Pilo, D. A. Rayner and A. Riotto, arXiv:hep-ph/0302087

[14] N. Arkani-Hamed, H. C. Cheng, P. Creminelli and L. Randall, Phys. Rev. Lett. 90, 221302 (2003).

[15] E. G. Adelberger [EOT-WASH Group Collaboration], arXiv:hep-ex/0202008

[16] S. Cullen and M. Perelstein, Phys. Rev. Lett. 83, 268 (1999); for a recent update, see: S. Hannestad and G. G. Raffelt, Phys. Rev. D 67, 125008 (2003);

[17] N. Arkani-Hamed, S. Dimopoulos and G. R. Dvali, Phys. Rev. D 59, 086004 (1999).

[18] P. Binetruy, C. Deffayet and D. Langlois, Nucl. Phys. B 565, 269 (2000); P. Kanti, I. I. Kogan, K. A. Olive and M. Pospelov, Phys. Lett. B 468 (1999) 31; C. Csaki, M. Graesser, L. Randall and J. Terning, Phys. Rev. D 62 (2000) 045015. 
[19] C. M. Will, Living Rev. Rel. 4 (2001) 4.

[20] A. Albrecht, C. P. Burgess, F. Ravndal and C. Skordis, Phys. Rev. D 65, 123507 (2002).

[21] E. Cremmer and J. Scherk, Nucl. Phys. B 108, 409 (1976); R. Sundrum, Phys. Rev. D 59, 085010 (1999); N. Arkani-Hamed, S. Dimopoulos and J. March-Russell, Phys. Rev. D 63, 064020 (2001).

[22] P. A. Dirac, Phys. Rev. 74, 817 (1948).

[23] N. Arkani-Hamed, L. J. Hall, D. R. Smith and N. Weiner, Phys. Rev. D 62, 105002 (2000); Z. Chacko, P. J. Fox, A. E. Nelson and N. Weiner, JHEP 0203, 001 (2002); A. Albrecht, C. P. Burgess, F. Ravndal and C. Skordis, Phys. Rev. D 65, 123506 (2002).

[24] K. R. Dienes, Phys. Rev. Lett. 88, 011601 (2002).

[25] E. Ponton and E. Poppitz, JHEP 0106, 019 (2001).

[26] K. R. Dienes, E. Dudas and T. Gherghetta, Nucl. Phys. B 557, 25 (1999) arXiv:hep-ph/9811428; N. Arkani-Hamed, S. Dimopoulos, G. R. Dvali and J. March-Russell, Phys. Rev. D 65, 024032 (2002).

[27] L. J. Hall and D. R. Smith, Phys. Rev. D 60, 085008 (1999). 